\title{
ON THE MINIMAL INVARIANT SUBSPACES OF THE HYPERBOLIC COMPOSITION OPERATOR
}

\author{
VALENTIN MATACHE
}

(Communicated by Palle E. T. Jorgensen)

\begin{abstract}
The composition operator induced by a hyperbolic Möbius transform $\phi$ on the classical Hardy space $H^{2}$ is considered. It is known that the invariant subspace problem for Hilbert space operators is equivalent to the fact that all the minimal invariant subspaces of this operator are one- dimensional. In connection with that we try to decide by the properties of a given function $u$ in $H^{2}$ if the corresponding cyclic subspace is minimal or not. The main result is the following. If the radial limit of $u$ is continuously extendable at one of the fixed points of $\phi$ and its value at the point is nonzero, then the cyclic subspace generated by $u$ is minimal if and only if $u$ is constant.
\end{abstract}

\section{INTRODUCTION}

Composition operators appeared implicitly in some works dealing with classical mechanics. A systematic study of this class of operators began during 19661968 when Ryff published [5] and, independently of him with slightly different methods, Nordgren made a somewhat similar study in [2]. Ever since, these operators have enjoyed constant attention. A good account of them through 1978 is given in [3].

The departure point of this paper, reporting on some results about minimal invariant subspaces, has been [4]. We shall denote as customary by $\mathrm{H}^{2}$ the classical Hardy space of all functions analytic on the open unit disc $D$ having square-summable Taylor coefficients. We recall that the Möbius transforms are the functions of the form

$$
\phi(z)=e^{i \theta} \frac{z-\alpha}{1-\bar{\alpha} z}, \quad z \in D,
$$

for any unimodular $e^{i \theta}$ and any $\alpha$ in $D$. Those that have precisely two fixed points on the unit circle $T$ are called hyperbolic. There also exist parabolic and elliptic Möbius transforms. If $\phi$ is analytic and $\phi$ maps $D$ into $D$ then the operator defined by

$$
C_{\phi} f=f \circ \phi, \quad f \in \mathrm{H}^{2},
$$

is called the composition operator induced by $\phi$ and, according to [2], is a bounded Hilbert space operator. For any hyperbolic $\phi$ there is a Möbius transform $\omega$ such that $\omega^{-1} \circ \phi \circ \omega$ is hyperbolic with preassigned fixed points,

Received by the editors July 29, 1991 and, in revised form, March 10, 1992.

1991 Mathematics Subject Classification. Primary 47B38; Secondary 47A15. 
for example, 1 and -1 . Since obviously $C_{\omega^{-1} \circ \phi \circ \omega}=C_{\omega} C_{\phi} C_{\omega}^{-1}$, this operator is similar to $C_{\phi}$. Therefore we shall consider in the sequel the hyperbolic transform

$$
\phi(z)=\frac{2 z+1}{z+2}, \quad z \in D,
$$

with fixed points 1 and -1 and call $C_{\phi}$ "the hyperbolic composition operator". The authors of [4] prove that the assertion that any bounded Hilbert space operator acting on a separable complex Hilbert space of dimension larger than 1 has nontrivial invariant subspaces is equivalent to the fact that all the minimal invariant subspaces of $C_{\phi}$ are one-dimensional. A minimal invariant subspace is obviously an atom of the invariant subspace lattice. Needless to say, such subspaces need to be cyclic. It, therefore, seems interesting to ask whether one could decide by the properties of some $u$ in $\mathrm{H}^{2}$ if the cyclic subspace $K_{u}=\bigvee_{n \geq 0} C_{\phi}^{n} u$ is minimal invariant or not. In the sequel we shall give some partial answers to this question.

\section{SOME RESULTS}

Suppose $X$ is a Banach space and $T$ an invertible bounded linear operator on $X$. We recall that some subspace $M$ of $X$ is called doubly invariant for $T$ if it is invariant both for $T$ and $T^{-1}$. We begin with the following interesting simple property of minimal invariant subspaces.

Theorem 1. Any minimal invariant subspace of $T$ is doubly invariant.

Proof. Suppose $M$ is a minimal invariant subspace of $T$. Then $M$ is cyclic, so there is $x$ in $X$ such that $M=\bigvee_{n \geq 0} T^{n} x$. Since $M$ is minimal, any nonzero vector in $M$, including $T x$, is cyclic. For any integer $n \geq 1, T^{-1}\left(T^{n} x\right)=$ $T^{n-1} x$ is in $M$ and $M=\bigvee_{n \geq 1} T^{n} x$ since $T x$ is cyclic. It follows that $M$ is invariant for $T^{-1}$.

We continue by clarifying some notation and simple calculations that we will use. By $\phi^{(n)}$ we denote $\phi \circ \phi \circ \cdots \circ \phi n$ times for any positive integer $n$. $\phi^{(0)}(z)=z$ for any $z \in D . \phi^{(-n)}$ is $\phi^{-1} \circ \cdots \circ \phi^{-1} n$ times. One can easily verify that

$$
\phi^{(n)}(z)=\frac{x_{n} z-y_{n}}{x_{n}-y_{n} z}, \quad \phi^{(-n)}(z)=\frac{x_{n} z+y_{n}}{x_{n}+y_{n} z}, \quad n=1,2,3, \ldots,
$$

where $x_{1}=2, y_{1}=-1$ and

$$
x_{n+1}=2 x_{n}-y_{n}, \quad y_{n+1}=2 y_{n}-x_{n}, \quad n=1,2,3, \ldots \text {; }
$$

hence,

$$
x_{n}=\left(3^{n}+1\right) / 2, \quad n=1,2,3, \ldots .
$$

We denote $z_{n}=\phi^{(n)}(0)$ for any integer $n$. For positive $n, z_{n}=-y_{n} / x_{n}$ and $z_{n} \rightarrow 1$ whereas $z_{-n}=-z_{n}, n=1,2,3, \ldots$.

Theorem 2. If $\alpha$ is one of the fixed points of $\phi, u\left(e^{i \theta}\right)$ is continuously extendable at $\alpha$, and $u(\alpha) \neq 0$, then $K_{u}$ is minimal if and only if $u$ is constant.

Proof. First suppose $\alpha=1$. Denote by $C$ the space of constant functions. For $0<r<1$ and $t$ in $[-\pi, \pi]$,

$$
P_{r}(t)=\frac{1-r^{2}}{1-2 r \cos t+r^{2}}
$$


is the Poisson kernel. The author of [2] observes that, for any $f$ in $L_{T}^{2}$, the following hold:

$$
\begin{aligned}
& \frac{1}{2 \pi} \int_{-\pi}^{\pi}(f \circ \phi)\left(e^{i \theta}\right) d \theta=\frac{1}{2 \pi} \int_{-\pi}^{\pi} f\left(e^{i \theta}\right) P_{|\phi(0)|}(\theta) d \theta \\
&\left\|C_{\phi}^{n} u-u(1)\right\|_{2}^{2}=\frac{1}{2 \pi} \int_{-\pi}^{\pi}\left|u\left(\phi^{(n)}\left(e^{i \theta}\right)\right)-u(1)\right|^{2} d \theta \\
&=\frac{1}{2 \pi} \int_{-\pi}^{\pi}\left|(u-u(1)) \circ \phi^{(n)}\left(e^{i \theta}\right)\right|^{2} d \theta \\
&=\frac{1}{2 \pi} \int_{-\pi}^{\pi}\left|u\left(e^{i \theta}\right)-u(1)\right|^{2} P_{z_{n}}(\theta) d \theta
\end{aligned}
$$

For arbitrary fixed $\varepsilon>0$ choose $\delta>0$ such that $\left|u\left(e^{i \theta}\right)-u(1)\right|^{2}<\varepsilon / 2$ if $|\theta| \leq \delta$, which is possible by the continuity assumption that we made on $u$. Observe that $P_{z_{n}}(\theta) \leq P_{z_{n}}(\delta)$ if $|\theta|>\delta$ and $P_{z_{n}}(\delta) \rightarrow 0$, so we may choose a positive integer $N$ such that $P_{z_{n}}(\delta)\|u-u(1)\|_{2}^{2}<\varepsilon / 2$ if $n \geq N$. We deduce that

$$
\begin{aligned}
\left\|C_{\phi}^{n} u-u(1)\right\|_{2}^{2}= & \frac{1}{2 \pi} \int_{-\delta}^{\delta}\left|u\left(e^{i \theta}\right)-u(1)\right|^{2} P_{z_{n}}(\theta) d \theta \\
& +\frac{1}{2 \pi} \int_{|\theta|>\delta}\left|u\left(e^{i \theta}\right)-u(1)\right|^{2} P_{z_{n}}(\theta) d \theta \\
\leq & \frac{\varepsilon}{2}+P_{z_{n}}(\delta)\|u-u(1)\|_{2}^{2}<\varepsilon
\end{aligned}
$$

if $n \geq N$. We proved that $\left(C_{\phi}^{n} u\right)_{n}$ tends to $u(1)$ in $\mathrm{H}^{2}$, and since $u(1) \neq 0$, we deduce $C \subset K_{u} . C$ is obviously invariant for any composition operator. If $u$ is nonconstant then $K_{u}$ is nonminimal. The conclusion of the theorem is now obvious.

For $\alpha=-1$ consider the transform $\omega(z)=-z, z \in D$. We have that $\omega=\omega^{-1}$ and $\omega \circ \phi^{(-1)} \circ \omega=\phi$. Consequently $\phi^{(n)}=\omega \circ \phi^{(-n)} \circ \omega$. If $v=u \circ \omega$ then $u \circ \phi^{(n)}=C_{\omega} v \circ \phi^{(-n)}$. Since $v(z)=u(-z)$ and $u\left(e^{i \theta}\right)$ is continuously extendable at $-1, v\left(e^{i \theta}\right)$ is continuously extendable at 1 . In the argument above replace $\phi$ by $\phi^{-1}$ and $u$ by $v$ and deduce $v \circ \phi^{(-n)} \rightarrow v(1)=u(-1) \neq 0$ in $\mathrm{H}^{2}$. Hence $u \circ \phi^{(n)}$ tends to $C_{\omega} u(-1)=(u(-1) \circ \omega)=u(-1)$. Once more $C \subseteq K_{u}$. The proof is complete.

Theorem 3. If $u$ is inner and $v$ is the greatest common inner divisor of the family $\left\{u \circ \phi^{(n)}, n=\ldots,-2,-1,0,1,2, \ldots\right\}$, then if $K_{u}$ is minimal $v$ is an eigenvector of $C_{\phi}$.

Proof. We denote by $v_{n}$ the greatest common inner divisor of the family $\{u \circ$ $\left.\phi^{(k)}, k \geq n\right\}$ for any integer $n$. If $K_{u}$ is minimal then $v_{n}=e^{i \theta_{n}} v$, for in this case

$$
K_{u}=\bigvee_{-\infty}^{\infty}\left(u \circ \phi^{(n)}\right)=\bigvee_{k \geq n}\left(u \circ \phi^{(k)}\right)
$$

for any fixed integer $n$.

So $K_{u} \subseteq v_{n} \mathrm{H}^{2}$ and hence $v_{n}$ divides $u \circ \phi^{(k)}$ for any integer $k$ and, consequently, $v_{n}$ divides $v$. Since obviously $v$ divides $v_{n}$, we obtain that 
$v=e^{i \theta_{n}} v_{n}$ for some unimodular $e^{i \theta_{n}}$. Now $u=v v_{0}$ for some inner $v_{0}$. This implies $u \circ \phi=(v \circ \phi)\left(v_{0} \circ \phi\right)$, hence $u \circ \phi \in(v \circ \phi) \mathrm{H}^{2} . u \circ \phi=v v_{1}$ for some inner $v_{1}$, hence $u \circ \phi^{(2)} \in(v \circ \phi) \mathrm{H}^{2}$. In this manner we obtain $K_{u} \subseteq(v \circ \phi) \mathrm{H}^{2}$. In the same way, $K_{u} \subseteq\left(v \circ \phi^{(n)}\right) \mathrm{H}^{2}$ for any integer $n$; hence, $K_{u} \subseteq \bigcap_{-\infty}^{\infty}\left(v \circ \phi^{(n)}\right) \mathrm{H}^{2}$. Since the intersection above is nonzero and invariant to the unilateral shift, Beurling's theorem implies that $\bigcap_{-\infty}^{\infty}\left(v \circ \phi^{(n)}\right) \mathrm{H}^{2}=v^{\prime} \mathrm{H}^{2}$ for some inner $v^{\prime} . v^{\prime} \mathrm{H}^{2} \subseteq v \mathrm{H}^{2}$ implies $v$ divides $v^{\prime} . K_{u} \subseteq v^{\prime} \mathrm{H}^{2}$ implies $v^{\prime}$ divides $v$. Hence $v \mathrm{H}^{2}=\bigcap_{-\infty}^{\infty}\left(v \circ \phi^{(n)}\right) \mathrm{H}^{2}$, if $K_{u}$ is minimal invariant. There are then $w^{\prime}, w^{\prime \prime}$ inner such that $v=(v \circ \phi) w^{\prime}$ and $v=\left(v \circ \phi^{-1}\right) w^{\prime \prime}$. So $v \circ \phi=v\left(w^{\prime \prime} \circ \phi\right)$. We see that $v$ divides $v \circ \phi$ and $v \circ \phi$ divides $v$. It follows that $v$ is an eigenvector of $C_{\phi}$.

Actually the proof of the previous theorem gives more information than stated; namely, $v_{n}$ could replace $v$ in the statement.

Theorem 4. If $u$ is inner and there is a sequence $\left(\lambda_{n}\right)_{n}$ of unimodular constants such that $\prod_{n \geq 0}\left(\lambda_{n} u \circ \phi^{(n)}\right)=v$ converges uniformly on compact subsets of $D$ and $v(0) \neq 0$, then $K_{u}$ is minimal invariant if and only if $u$ is constant.

Proof. If $v_{n}=\prod_{k \geq n} \lambda_{k} u \circ \phi^{(k)}$ then $\left\|v_{n}\right\|_{\infty} \leq 1$. Consequently

$$
\left\|v_{n}-1\right\|_{2}^{2}=\left\langle v_{n}, v_{n}\right\rangle+\langle 1,1\rangle-2 \operatorname{Re}\left\langle v_{n}, 1\right\rangle \leq 2\left(1-\operatorname{Re} \prod_{k \geq n} \lambda_{k} u\left(z_{k}\right)\right) \text {. }
$$

$v(0) \neq 0$ implies $\left\|v_{n}-1\right\|_{2} \rightarrow 0$, hence $v_{n} \rightarrow 1$ in $\mathrm{H}^{2}$. For any $h$ in $\mathrm{H}^{2}$, $\left\langle z v_{n} h, \lambda_{k} u \circ \phi^{(k)}\right\rangle=\left\langle z h v_{n} / \lambda_{k} u \circ \phi^{(k)}, 1\right\rangle=0$ if $k \geq n$. Suppose $K_{u}$ is minimal. Then $\bigvee_{k \geq n}\left(u \circ \phi^{(k)}\right)=K_{u}$ for any $n$. It follows that $z v_{n} \mathrm{H}^{2} \subseteq \mathrm{H}^{2} \ominus K_{u}$ for any $n$. Hence $z \mathrm{H}^{2} \subseteq \mathrm{H}^{2} \ominus K_{u}$ and so $K_{u} \subseteq \mathrm{H}^{2} \ominus z \mathrm{H}^{2}=C$. The result follows.

Application. One could derive a sufficient condition for Blaschke products; namely, if $B$ is a Blaschke product with infinitely many zeros $\left(\alpha_{n}\right)_{n}$ and if

is finite and

$$
\sum_{n, k \geq 0} 1-\left|\phi^{(-k)}\left(\alpha_{n}\right)\right|^{2}
$$

then $K_{B}$ is nonminimal.

Proof. Denote $\phi_{\alpha}(z)=(z-\alpha) /(1-\bar{\alpha} z)$ for any $\alpha$ in $D$. We observe that $\phi_{\alpha_{n}} \circ \phi=\phi_{\phi^{-1}\left(\alpha_{n}\right)} c_{n}$ where $\left|c_{n}\right|=1$. Therefore if $B_{1}$ is the Blaschke product of zeros $\left(\phi^{-1}\left(\alpha_{k}\right)\right)_{k}$, then there is no problem to see that $B \circ \phi=e^{i \theta_{1}} B_{1}$ for some unimodular $e^{i \theta_{1}}$. Just as above, $B \circ \phi^{(k)}=e^{i \theta_{k}} B_{k}$ where $B_{k}$ is the Blaschke product of zeros $\left(\phi^{(-k)}\left(\alpha_{n}\right)\right)_{n}$. Taking $\lambda_{k}=e^{-i \theta_{k}}$ and $u=B$, one obtains in Theorem 4 the Blaschke product of zeros $\left(\phi^{(-k)}\left(\alpha_{n}\right)\right)_{k, n}$, which is convergent if (6) holds and has a nonzero value at 0 if (7) holds.

\section{QUESTIONS AND COMMENTS}

Question 1. If $u$ is in $\mathrm{H}^{2}, u(1)=u(-1)=0$, and $u$ satisfies the good continuity condition "around" one of the fixed points, is it possible to deduce that $K_{u}$ is not minimal? 
Comments. There is some hope to be so. At any rate if one takes $u(z)=z^{2}-1$, which is continuous on $T$, and $u(1)=u(-1)=0$, one can prove that still $K_{u}$ is nonminimal. Indeed if we suppose it is minimal, note that $K_{u} \subseteq \bigvee_{k \geq n}\left(\phi^{(k)}\right)^{2}+C$ for any $n$. If $B_{n}=\prod_{k \geq n}\left(\phi^{(k)}\right)^{2}$, we observe that $z B_{n} \mathrm{H}^{2}$ is orthogonal to any $\left(\phi^{(k)}\right)^{2}$ for $k \geq n$. So $\bigvee_{k \geq n}\left(\phi^{(k)}\right)^{2} \subseteq \mathrm{H}^{2} \ominus z B_{n} \mathrm{H}^{2}$ for any $n$. Hence $K_{u} \subseteq \bigcap_{n \geq 0}\left(\mathrm{H}^{2} \ominus z B_{n} \mathrm{H}^{2}\right)=C$ because $\left\|B_{n}-1\right\|_{2}^{2}=2\left(1-\operatorname{Re} B_{n}(0)\right) \rightarrow 0$ and, hence, $\left(\bigcap_{n \geq 0}\left(\mathrm{H}^{2} \ominus z B_{n} \mathrm{H}^{2}\right)\right)^{\perp}=\bigvee_{n \geq 0} z B_{n} \mathrm{H}^{2}=z \mathrm{H}^{2}$. Hence $K_{u} \subseteq C$, which is absurd. So $K_{u}$ is nonminial.

On the other hand, the method we use in Theorem 2 is ineffective here. Take, for instance, $u(z)=\left(z^{2}-1\right) B$ where $B$ is the Blaschke product of zeros $\left(z_{k}\right)_{k \geq 1}$. Obviously $u$ is continuous on $T, u(1)=u(-1)=0$, and $\left\langle u \circ \phi^{(k)}, 1\right\rangle=u\left(z_{k}\right)=0$ for any $k \geq 1$. Hence $C \nsubseteq K_{u}$.

Question 2. For inner functions Theorems 3 and 4 give some rather particular tricks to deduce $K_{u}$ is not minimal even when $u$ has no continuous extensions at 1 or -1 . Is it possible to obtain a general condition for inner functions?

Question 3. Is it possible to obtain implications of type " $u=f g$ and if $K_{f}$ is nonminimal then $K_{u}$ is nonminimal"?

Comments. This seems to be hard, however, it is tempting to try since $\mathrm{H}^{2}$ functions have well-known canonical factorizations.

\section{ACKNOWLEDGMENT}

The author thanks the referee for many useful suggestions and constructive criticism.

\section{REFERENCES}

1. K. Hofman, Banach spaces of analytic functions, Prentice-Hall, Englewood Cliffs, NJ, 1962.

2. E. A. Nordgren, Composition operators, Canad. J. Math. 20 (1968), 442-449.

3. _ Composition operators in Hilbert spaces, Hilbert Space Operators, Lecture Notes in Math., vol. 693, Springer Verlag, Berlin, Heidelberg, and New York, 1978, pp. 37-63.

4. E. A. Nordgren, P. Rosenthal, and F. S. Wintrobe, Invertible composition operators on $H^{p}$, J. Funct. Anal. 73 (1987), 324-344.

5. J. V. Ryff, Subordinate $H^{p}$-functions, Duke Math. J. 33 (1966), 347-354.

University of Timisoara, Department of Mathematics, Bv. Vasile Parvan 4, 1900 TIMISOARA, ROMANIA 